\title{
Die palmare Plattenosteosynthese bei distaler Radiusfraktur - Indikation und Technik
}

\author{
Torsten Pabst, Torsten Uzdil, Karl Heinrich Winker
}

\section{Zusammenfassung}

Der radio-palmare Zugang zum distalen Radius ist komplikationsarm. Die palmare Plattenosteosynthese des distalen Radius stellt die Methode der Wahl bei Flexionsfrakturen mit der Möglichkeit der sofortigen frühfunktionellen Nachbehandlung dar. Die Versorgung von Extensionsfrakturen - auch mit höhergradiger Instabilität - wird heute durch graziles und winkelstabiles Plattendesign ermöglicht. Auch hier ist die frühfunktionelle
Nachbehandlung bei ausreichender Knochenqualität zum Standard geworden. Längerdauernde Gipsruhigstellungen sind nur noch bei höhergradiger Osteoporose oder komplexer Frakturkonfiguration angezeigt. Somit wird die Gefahr einer bleibenden Funktionsstörung der betroffenen Hand deutlich reduziert, eine sympathische Reflexdystrophie weitestgehend ausgeschlossen. Die Entfernung des Osteosynthesematerials ist nur in wenigen Ausnahmefällen indiziert.

Unerlässlich ist die Beurteilung der neurologischen Situation der betroffenen Hand, insbesondere muss das etwaige Vorliegen einer frakturbedingten Alteration des N. medianus sicher beurteilt werden (Abb.1). ten Frakturlokalisationen dar. Der klassische Unfallmechanismus der distalen Radiusfraktur ist der Sturz auf die ausgestreckte Hand, meist in dorsalflektierter Stellung. Hierbei resultiert eine metaphysäre Frakturlokalisation (,in loco typico“) mit nach dorsal gerichteter Abkippung der radio-karpalen Gelenkfläche nach Colles [1]. Bei den selteneren Sturzereignissen auf die palmarflektierte Hand resultiert eine Flexionsfehlstellung ( $\mathrm{n}$. Smith).

\section{Diagnostik}

Bei der klinischen Untersuchung der distalen Radiusfraktur lässt sich neben den klassischen Frakturzeichen meist eine je nach dem Frakturmechanismus mehr oder weniger deutliche Fehlstellung des Handgelenkes nachweisen.

OP-JOURNAL 2003; 19: 42-50

(c) Georg Thieme Verlag Stuttgart · New York
Abb.1 Konventionelle Röntgenaufnahmen des Handgelenkes in 2 Ebenen.

\section{Therapie}

Die Behandlung der instabilen Frakturen mit oder ohne Beteiligung des radio-karpalen und/oder des distalen radioulnaren Gelenkes (DRUG) war lange Zeit die uneingeschränkte Domäne der konservativen und semikonservativen Therapieoptionen mit Retention im Fixateur externe bzw. im Gipsverband nach erfolgter Reposition, eventuell in Kombination mit dem Einbringen von Kirschner-Drähten, um das Repositionsergebnis des radiokarpalen Blockes zu sichern.

Folgen dieser mehrwöchigen Ruhigstellung waren nicht nur ein deutlich verminderter Kalksalzgehalt des gesamten Vorderarmes, nicht selten kam es zu erheblichen resultierenden Bewegungseinschränkungen des betroffenen Handgelenkes oder sogar zum Bild der sympathischen Reflexdystrophie, was eine langwierige Rehabilitationsphase, mitunter mit bleibenden erheblichen Funktionsstörungen der betroffenen Hand zur Folge hatte. Aufgrund dieser zum Teil schlechten Ergebnisse erwuchs die Forderung nach der Möglichkeit der frühfunktionellen Nachbehandlung.

\section{Indikation zur palmaren Plattenosteosynthese}

In Kenntnis des Umstandes, dass in der Regel nicht die Reposition der Fraktur, sondern die exakte Retention das entscheidende therapeutische Problem darstellt, ist in den letzten Jahren ein Wandel in der Strategie der Behandlung der distalen Radiusfrakturen eingetreten [3].

Nachdem sich bei den distalen Radiusfrakturen vom Flexionstyp (B3) die offene Reposition und palmare Plattenosteosynthese im Sinne einer echten Abstützplatte mit der Möglichkeit der sofortigen funktionellen Nachbehandlung etabliert hatte, gestaltete sich dieses Vorgehen bei der weitaus häufigeren Extensionsfraktur (A2, A3, C1, C2) mit den konven- 


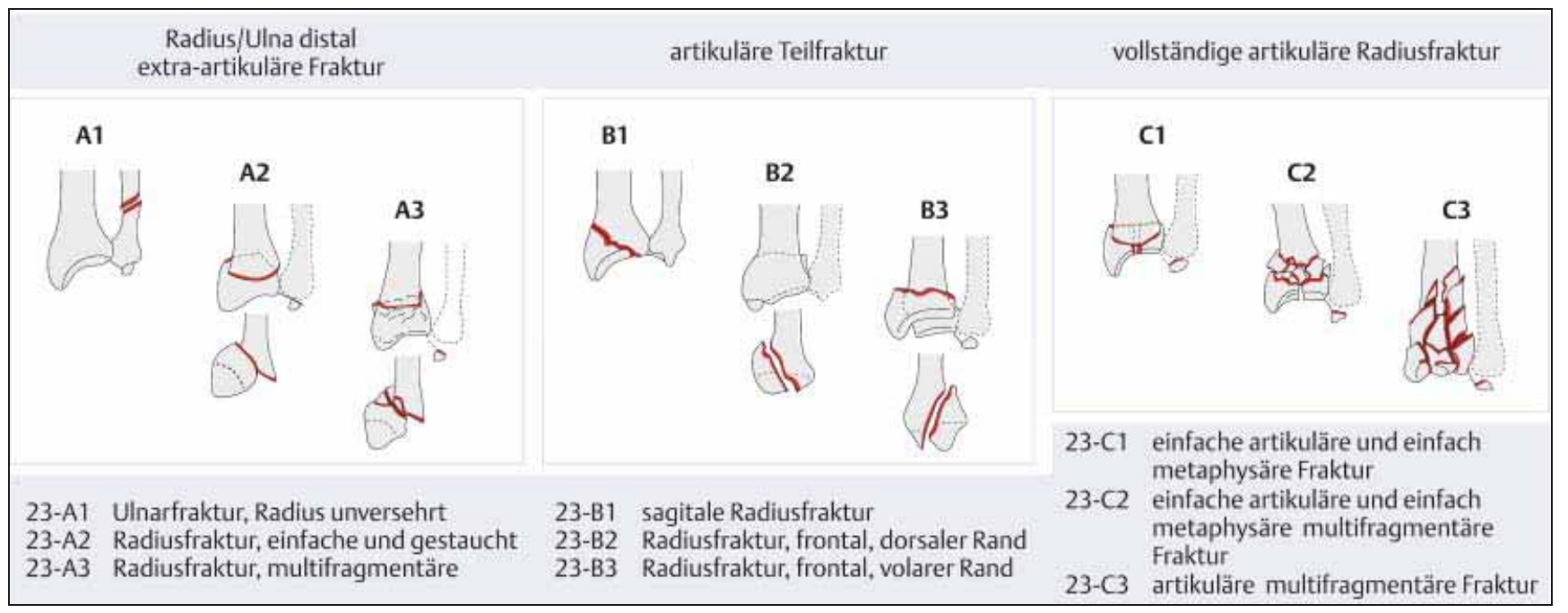

Abb. 2 AO-Klassifikation der distalen Unterarmfrakturen.

tionellen Plattendesignmerkmalen und der daraus potentiell bestehenden Gefahr der Re-Dislokation insbesondere im Sinne der Abkippung des distalen Fragmentes schwierig.

Eine Lösung dieses Problems wurde durch Kombination von palmarer Plattenosteosynthese mit Auffüllung des metaphysären Defektes mit kortikospongiösen Beckenkammspänen gesucht [4].

Bei intakter palmarer Kortikalis gewann seit 1995 die alleinige palmare Plattenosteosynthese, zunächst noch mit konventionellen - nicht winkelstabilen Platten, an Bedeutung. Hier machte man sich das Zuggurtungsprinzip zu Nutzen. Dieses Verfahren kam vorzugsweise A3- und C1-Frakturen mit der Möglichkeit der festen Verankerung der Schrauben zur Anwendung [5].

Erst durch die neue Generation der Osteosyntheseplatten mit der Möglichkeit der winkelstabilen Verankerung von Bolzen oder Schrauben gelang die erfolgreiche stabile Versorgung der instabilen Extensionsfrakturen von palmar mit der Möglichkeit einer hohen Primärstabilität. Durch graziles Plattendesign z. B. der palmaren „distalen Radiusplatte 2,4/ 2,7 mm“ der Firma Mathys/Synthes mit der Möglichkeit, im distalen Fragment bis zu 6 winkelstabile Bolzen oder 2,0mm-Schrauben einzubringen, gelingt heute auch die sichere Retention von distalen Radiusfrakturen mit hochgradiger Zerstörung der radialen Gelenkfläche (bis hin zur C3-Fraktur).
Im Hinblick auf das steigende durchschnittliche Lebensalter unserer Patienten und somit dem verstärkten Auftreten der Osteopenie/Osteoporose spielen die winkelstabilen Platten einen weiteren Vorteil der höheren Primärstabilität aus: das früher oftmals nachweisbare „Sintern" (= dorso-palmarer und axialer Korrekturverlust) der versorgten Extensionsfraktur nach Retention mit einer palmaren - konventionellen - Plattenosteosynthese kann durch das Einbringen von winkelstabilen Schrauben bzw. Bolzen in den auch bei Osteoporose stabilsten, unmittelbar subchondralen Knochen des distalen Radiusfragmentes vermieden werden [2]. Selbst bei hochgradiger Osteoporose ist hierdurch in den meisten Fällen nur eine kurzzeitige Ruhigstellung erforderlich.

Durch winkelstabile Implantate ist eine hohe Primärstabilität ohne Korrekturverlust bei der Versorgung instabiler Extensionsfrakturen selbst beim osteoporotischen Knochen zu erreichen.

\section{Operationszeitpunkt}

Offene Frakturen werden unverzüglich operativ versorgt. Bei distalen Radiusfrakturen am Mehrfachverletzten ist der Zeitpunkt der Operation abhängig vom Dislokations- und Instabilitätsgrad. Während bei geschlossener Fraktur und geringer Dislokation durchaus die Retention im gespaltenen Gipsverband als temporäre Maßnahme bis zur definitiven Versorgung der Fraktur ihre Bedeutung behalten hat, ist bei einer solchen Situation mit offenem Weichteilschaden oder komplexer Instabilität mit höhergradiger Destruktion des radialen Gelenkblockes die primäre Retention im handgelenkübergreifenden Fixateur externe angezeigt. Die definitive Versorgung kann dann nach Stabilisierung des Allgemeinzustandes des Patienten folgen.

Die Plattenosteosynthese der geschlossenen distalen Radiusfraktur mit tolerabler Fehlstellung ohne neurologische Symptomatik sollte frühzeitig innerhalb der ersten 12 bis 24 Stunden nach dem Unfall erfolgen, im Einzelfall kann die Versorgung frühelektiv bis zum Ende der ersten Woche terminiert werden. In einem solchen Fall wird die Fraktur nach schmerzlosem Aushang durch das Eigengewicht des Armes im „Mädchenfänger“ ohne zusätzliches Repositionsmanöver im gespaltenen zirkulären Unterarm-Gipsverband oder alternativ dazu mit einer Gipsschiene vorübergehend ruhiggestellt und die Extremität konsequent hochgelagert. Ein oft brüskes geschlossenes Repositionsmanöver, ggf. ohne suffiziente Analgesie, sollte unter allen Umständen vermieden werden, weil allein dieses Manöver den ersten Schritt zur Ausbildung einer sympathischen Reflexdystrophie darstellen kann (Abb.3a,b).

Liegt eine nicht tolerable Fehlstellung bei geschlossener Weichteilsituation ohne neurologische Symptome vor, sollten die Reposition der Fraktur und die simultane Osteosynthese frühelektiv, z.B. innerhalb der ersten 24 Stunden, folgen.

Bei traumabedingter Funktionsstörung des N. medianus ist die dringliche operative Dekompression des Nerven mit einzeitiger Versorgung der distalen Radiusfraktur indiziert. 

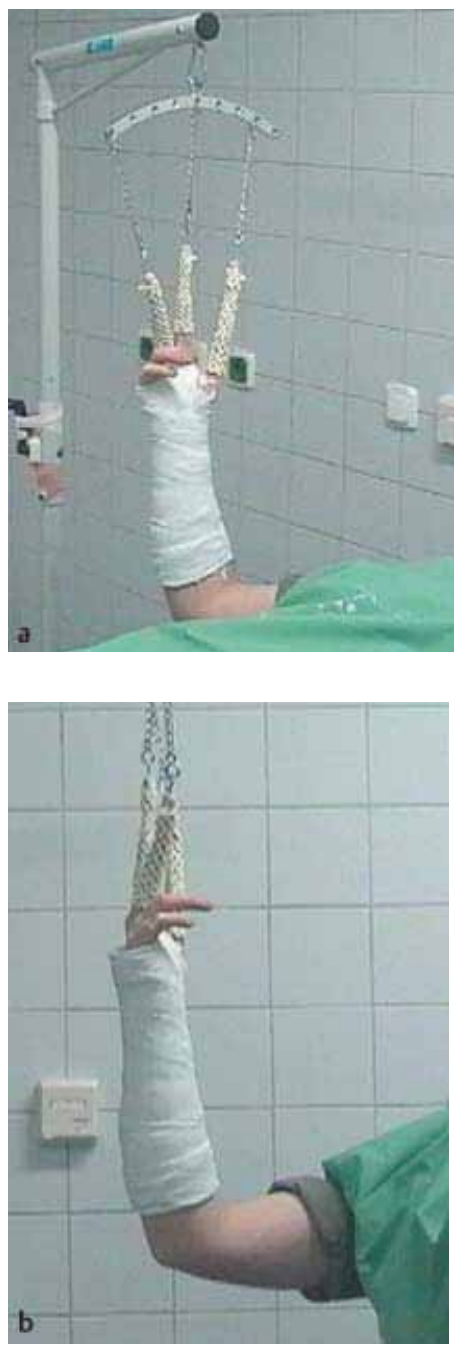

Abb.3au.b Gipsanlage im „Mädchenfänger“ ohne Repositionsmanöver.

Bei bestehender Operationsindikation keine Repositionsmanöver, sondern nur Ruhigstellung und Hochlagerung. Operative Versorgung in der Regel innerhalb von 12-24 h. Bei neurologischer Symptomatik sofortige Operation.

\section{Plattendesign}

Zur palmaren Plattenosteosynthese stehen Platten verschiedener Hersteller mit vielfältigen Designunterschieden zur Verfügung. Die meisten Platten haben eine T-Form (schräg oder gerade) und sind in Anpassung an die Wölbung der Palmarseite des distalen Radius bereits vom Hersteller mit einer entsprechenden Biegung versehen. Die Versorgung von Flexionsfrakturen erfolgt mit konventionellen 3,5-mm-T-Radius-Platten. Zur Versorgung von Extensionsfrakturen wurden diese zunächst ebenfalls verwendet, hier zeigte sich jedoch bei instabilen Fraktu-


Abb.4a-d Beispiele winkelstabiler Platten für den distalen Radius ((a) T-LCP $3,5 \mathrm{~mm}$, schräger und gerader T-Schenkel; (b) 3 vs. 6 distale Löcher; (c) 2,4/2,7-mm-Platte; (d) Locking-Distal-Radius-System 2,4, alles Titan, Synthes).

ren ein dorsoaxialer Korrekturverlust [2]. Eine frühfunktionelle Behandlung war nicht möglich.

Heute werden bevorzugt Platten mit der Möglichkeit der winkelstabilen Verankerung von Schrauben (z.B. T-LCP 3,5 mm, gerade bzw. schräg, Stahl oder Titan, Synthes) oder Bolzen (distale T-Radiusplatte 2,4/2,7 mm, Stahl oder Titan, Synthes) aus genannten Gründen zur Versorgung instabiler Extensionsfrakturen des distalen Radius verwendet. Die Richtung der winkelstabilen Verankerungselemente ist in einem Winkel von ca. $85^{\circ}$ zum distalen Plattenschenkel festgelegt. Neuerdings stehen aus der 2,4-mm-Plattengeneration der Locking-Compression-Serie für den distalen Radius eine gerade, eine Lförmige und eine T-förmige Platte zur Verfügung. Eigene Erfahrungen mit diesen neuen Implantaten liegen derzeit noch nicht vor.
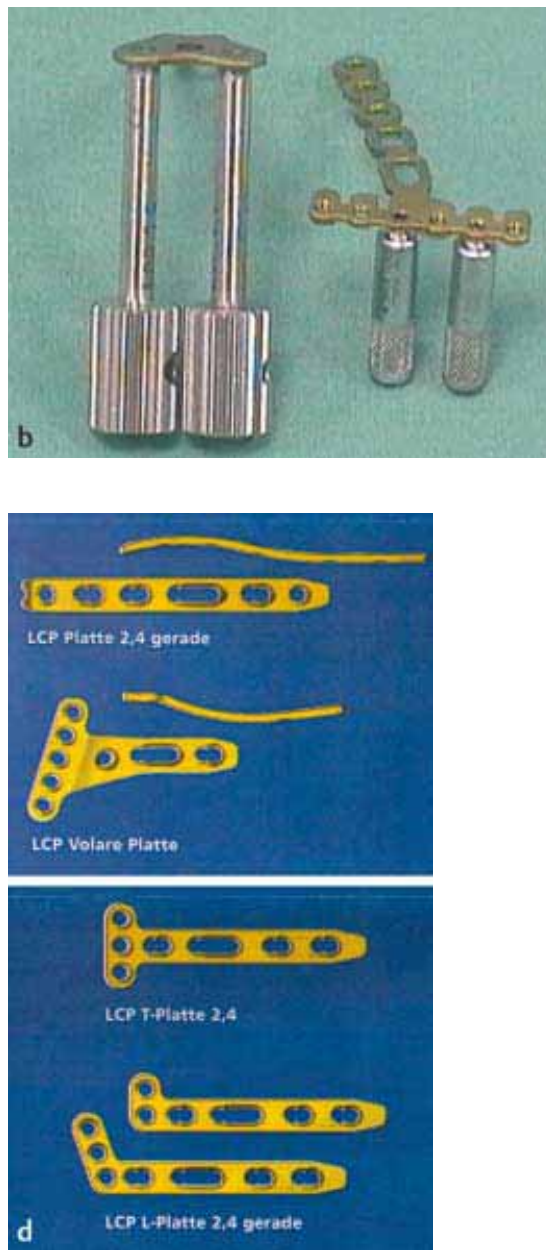

Wenige Plattenmodelle können ein breites Frakturspektrum abdecken (Abb.4a-d).

\section{Operatives Vorgehen}

\section{Narkose/Lagerung}

Der Eingriff erfolgt in pneumatischer Blutleere und kann sowohl in Regionalanästhesie als auch in Allgemeinanästhesie vorgenommen werden. Der Patient wird auf dem Rücken mit auf dem Armtisch ausgestrecktem Arm gelagert. Der Unterarm wird frei beweglich steril abgedeckt. Der Operateur sitzt im Winkel zwischen Körper und Arm des Patienten, der Assistent im Winkel zwischen Arm und Kopf. Der Bildverstärkerarm kann somit von distal her über die verletzte Extremität gefahren werden (Abb.5a,b). 
a, b

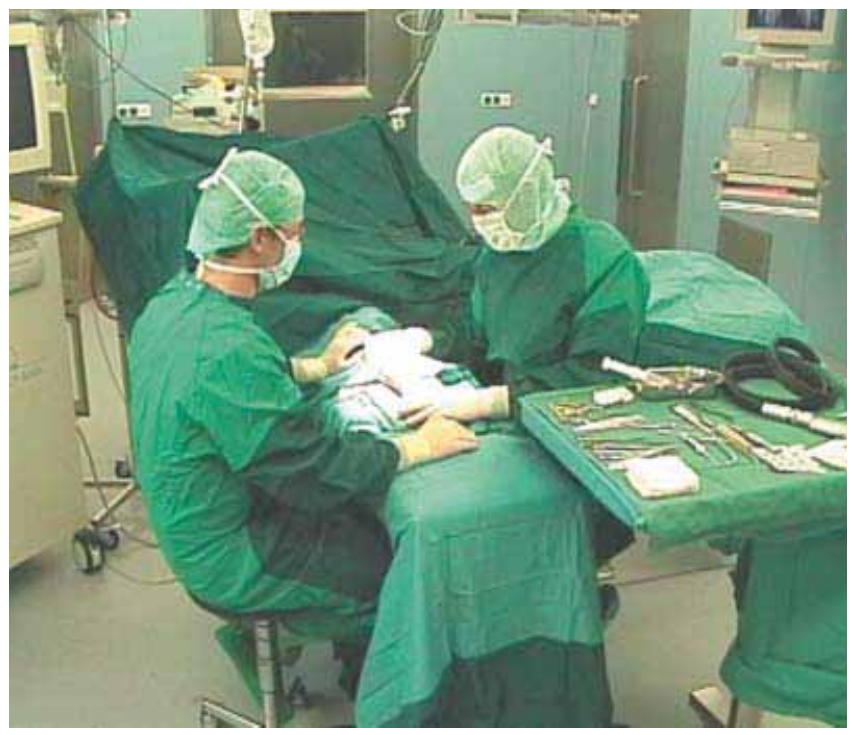

Abb.5au.b Lagerung/Operateurposition.
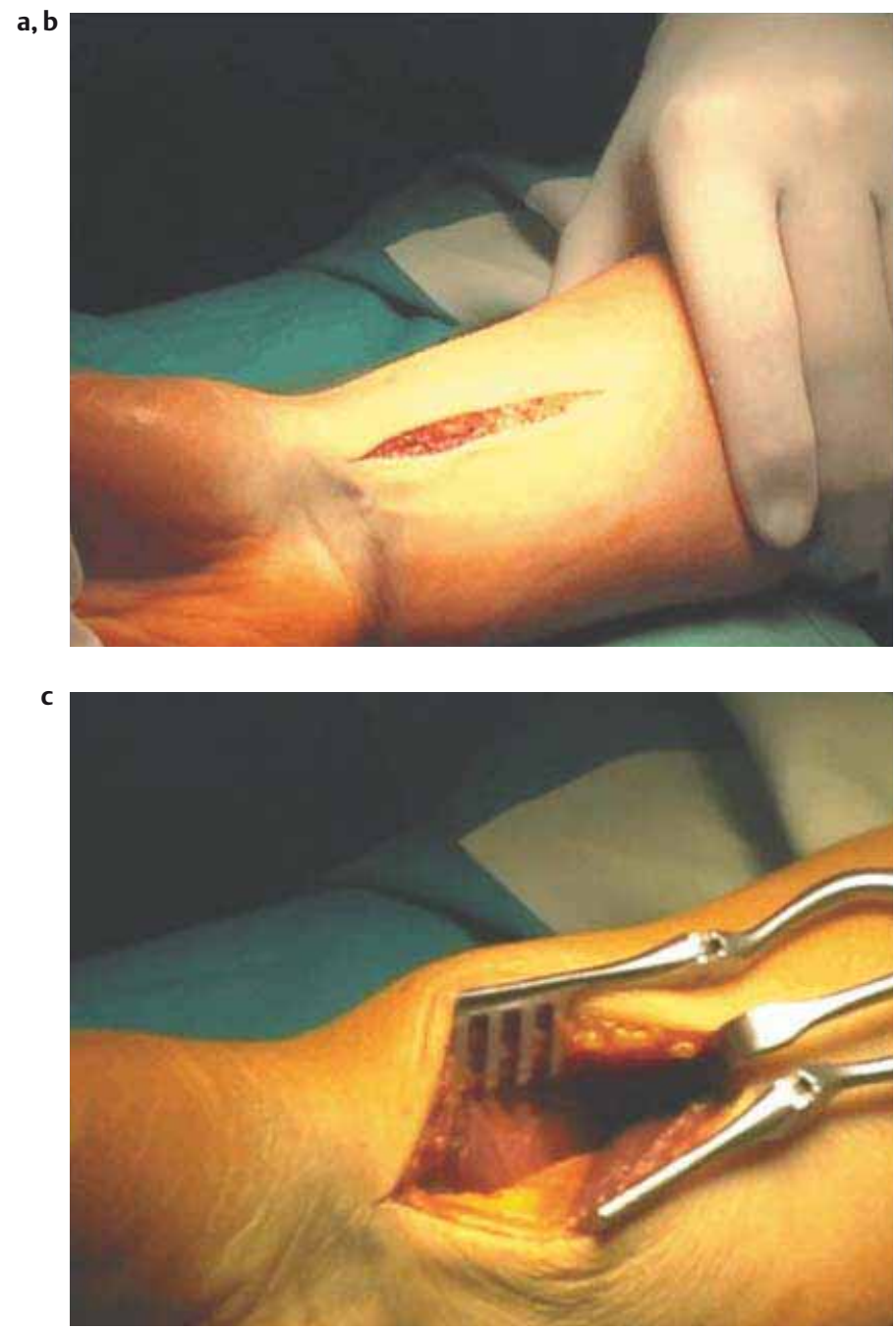
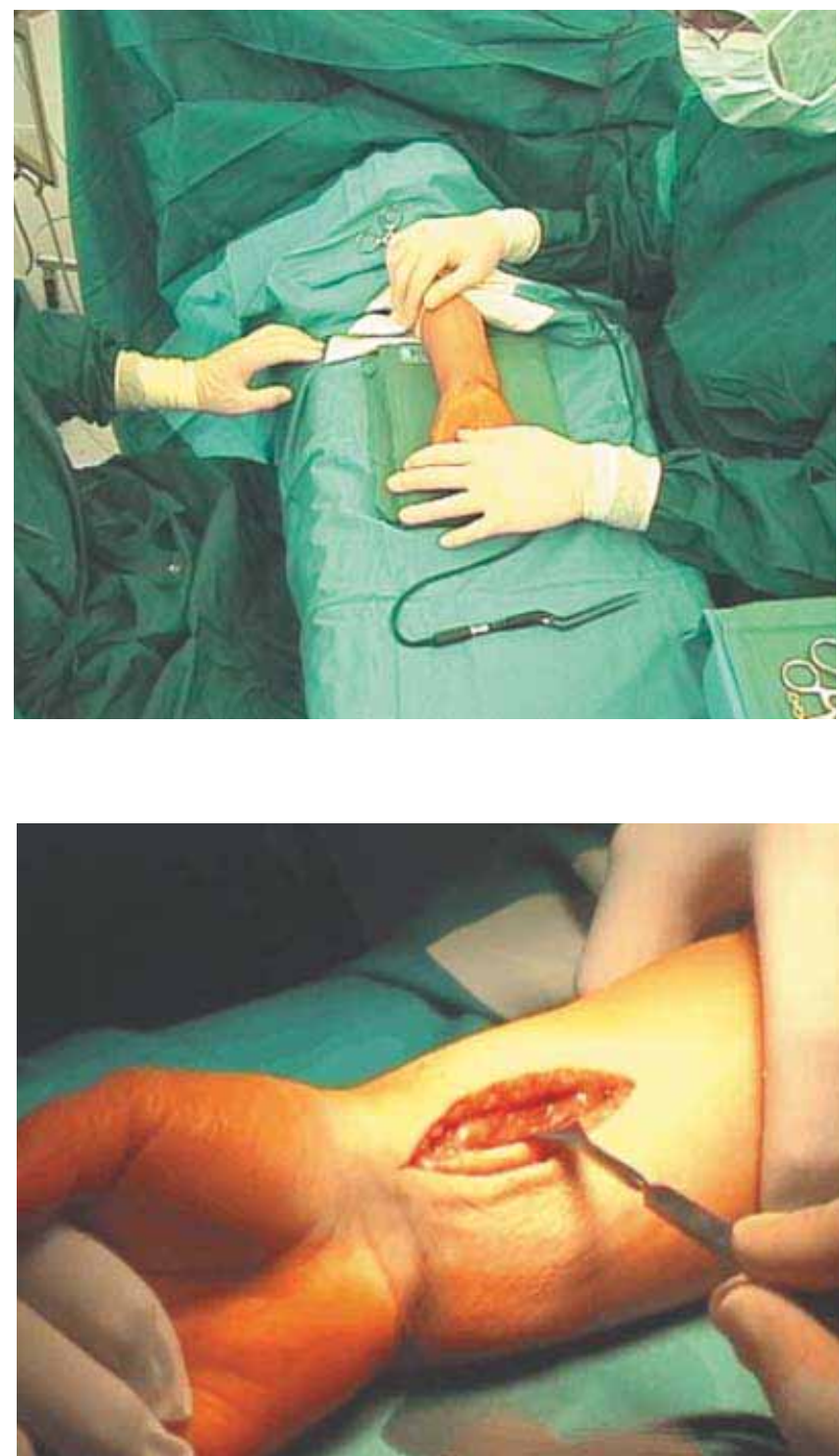

Abb.6a-c Radio-palmarer Zugang zum distalen Radius. 

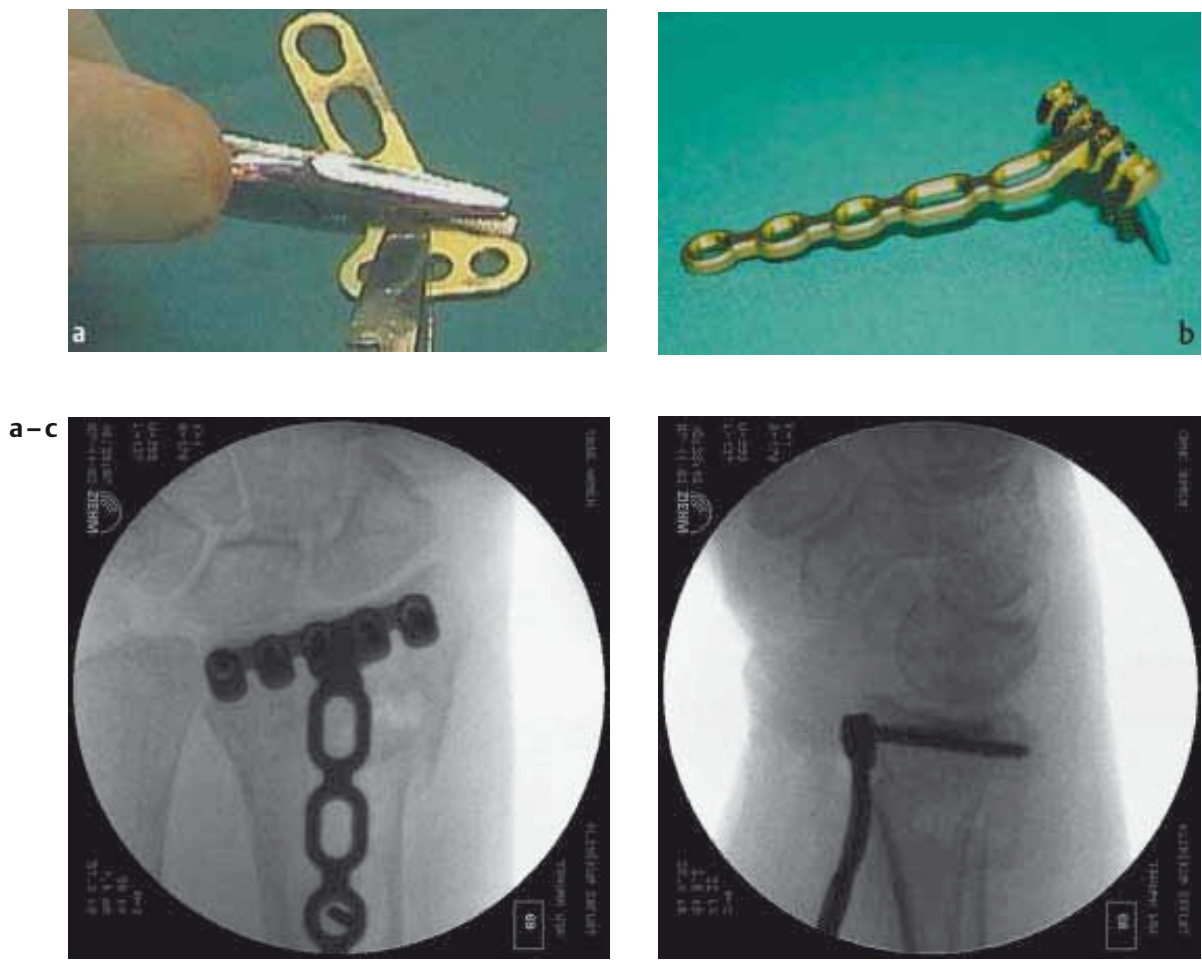

d

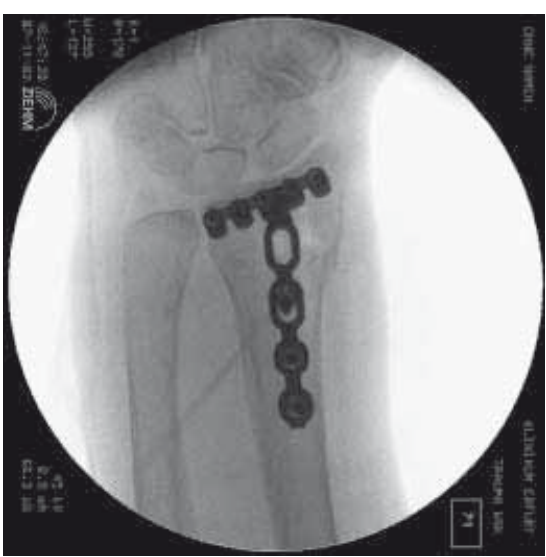

\section{Zugang/Anatomie}

Der von uns favorisierte radio-palmare Zugang zum distalen Radius radial der Sehne des M. flexor carpi radialis stellt einen komfortablen Zugang zur palmaren Plattenosteosynthese bei distalen Radiusfrakturen dar. Bei der Hautinzision sollte das Kreuzen der Handgelenksbeugefalten vermieden werden. Hierbei werden nach Präparation durch die Fascia antebrachii in Höhe der Sehnenscheide dieser Leitstruktur die A. radialis und durch Abschieben aller Finger-Beugesehnen nach ulnar der N. medianus inklusive des Ramus palmaris nervi mediani in aller Regel sicher geschont. Der M. pronator quadratus wird quer zur Faserrichtung im Bereich seines radialen Ansatzes durchtrennt. Die Beugeseite des distalen Radius stellt

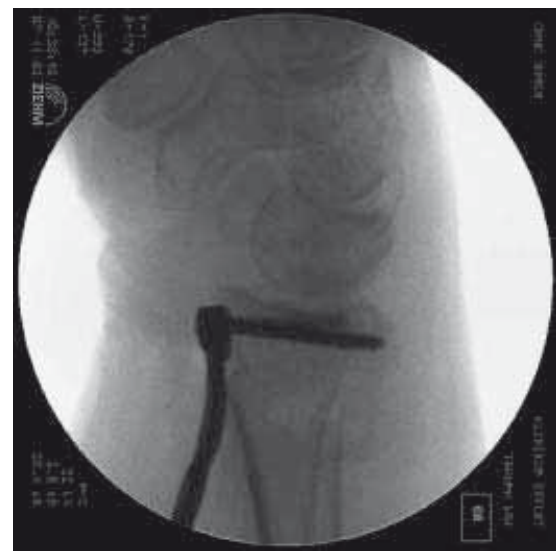

Abb. 8a-d Reposition mit Platte (Beispiel: 2,4/2,7-mm-Platte, Synthes).

sich nun konkav geformt dar, das distale Radiusende verbreitert sich zur Gelenkfläche hin. Lediglich bei Frakturen mit kleinem ulnaren Schlüsselfragment erfolgt der palmare Zugang ulnar der Sehnen des M. flexor carpi radialis, da so die Reposition und Fixierung dieses Frakturanteiles erleichtert ist.

Sollte eine primäre Entlastung des N. medianus erforderlich sein, erfolgt dies über einen separaten Zugang in der Hohlhand in Mini-release-Technik.

Der radiopalmare Zugang zum distalen Radius ist komfortabel und komplikationsarm. Die Operationstechnik kann rasch erlernt werden, der Eingriff ist eine „Assistentenoperation“ (Abb.6ac).
Abb.7au.b (a) Biegen der 3,5-mm-T-LCP mit schrägem T-Schenkel; (b) herstellerseitig definierter dorsopalmarer Winkel der Bolzen bei der 2,4/2,7-mm-Platte.

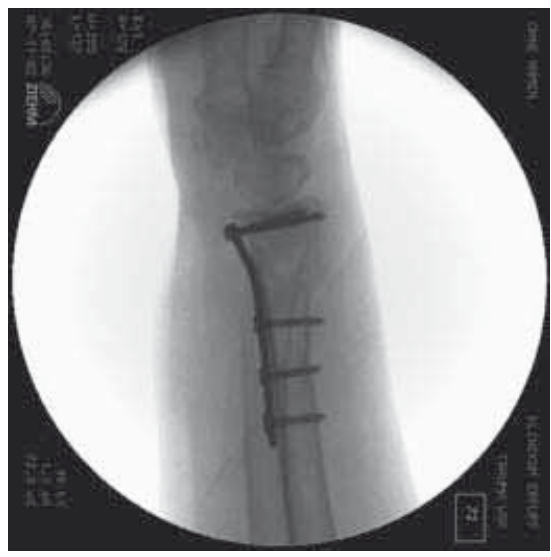

Alternativ wird von einigen Autoren der klassische palmare Zugang zum distalen Radius mit primärer Eröffnung des Karpaltunnels und Eingehen radial der Sehne des M. flexor carpi ulnaris favorisiert. Dieser besitzt jedoch aufgrund des Ausmaßes der Inzision mit Kreuzung der Handbeugefalte und der Retinacula flexorum lediglich nur noch eingeschränkte Bedeutung.

\section{Operationsablauf/Reposition}

Nach Darstellen der Fraktur und Säuberung des Frakturspaltes kann die Reposition auf zwei grundsätzlich verschiedenen Wegen erfolgen:

1. Direkte schrittweise Reposition der Fragmente unter Wiederherstellung der möglichst exakten Anatomie der Epi- und Metaphysenzone mit nachfolgender Retention durch Auflage der anmodellierten Platte und Fixierung durch die winkelstabilen Plattenschrauben

2. Exakte Reposition der Epiphyse des distalen Radius, winkelstabile Montage der angepassten Platte an das epiphysäre Fragment und Reposition der Fraktur durch Befestigen der Platte am meta- und diaphysären Fragment unter Ausnutzung des vorher festgelegten Plattenschaft-Winkels.

Der Vorteil beim letztgenannten Verfahren besteht darin, dass bei Hyperflexion 

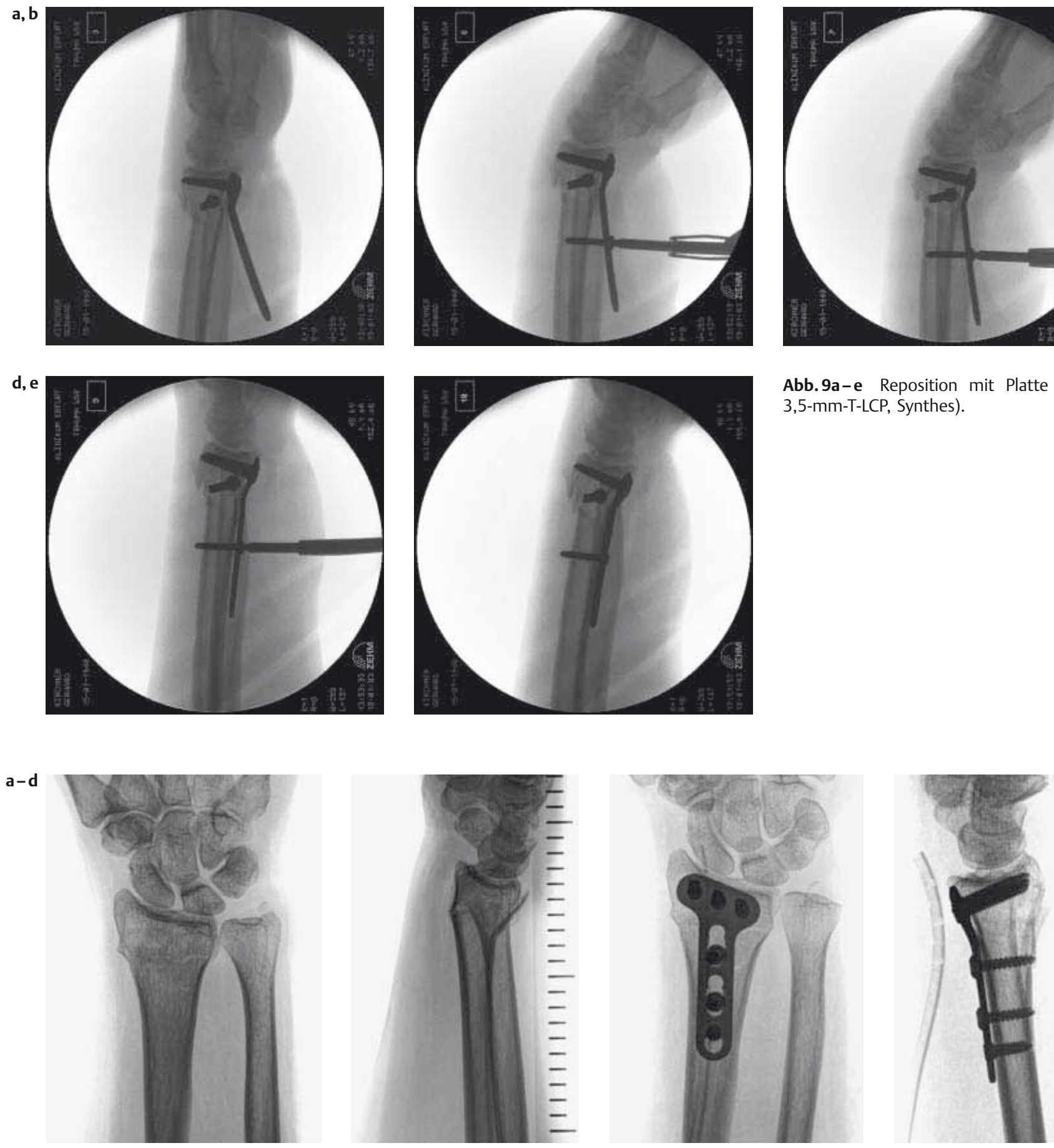

Abb.10a-d Versorgung einer A3-Fraktur mit 3,5-mm-T-LCP mit schrägem Schenkel.

im Handgelenk zunächst in der seitlichen Einstellung unter Bildverstärkersicht eine exakt subchondrale Lage der Verankerungselemente in der Zone der größten Knochenfestigkeit im distalen Fragment ermöglicht werden kann, so dass die Gefahr der sekundären Re-Dislokation selbst beim osteoporotischen Knochen minimiert ist.
Während bei der palmaren 2,4/2,7-mmPlatte und der 3,5-mm-T-LCP mit geradem T-Schenkel der dorsopalmare Neigungswinkel vom Hersteller bereits in Anpassung an die palmare Kontur des distalen Radius angelegt ist, muss bei Auswahl der 3,5-mm-T-LCP die manuelle Zurichtung der Platte erfolgen (Abb. $7 \mathbf{a}, \mathbf{b})$.

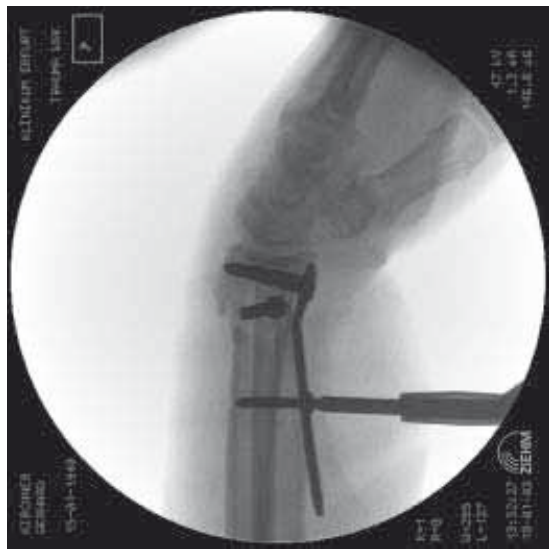

Abb.9a-e Reposition mit Platte (Beispiel 3,5-mm-T-LCP, Synthes).
Bei der Reposition ist besondere Beachtung der Wiederherstellung der Längenverhältnisse zwischen Radius und Ulna, der Beseitigung von Stufen in der radialen Gelenkfläche und auf den Ausgleich des meist vorhandenen Radialversatzes sowie Fehlrotation des distalen Fragmentes zu schenken. 




Im Falle des Vorliegens eines ulnaren Schlüsselfragmentes wird dieses ebenfalls über den distalen Plattenanteil mit fixiert (Abb. 8a-d, Abb. 9a-e, Abb. $10 a-d)$.

In Einzelfällen, insbesondere bei hochgradigen Instabilitäten (C3), kann die Unterstützung der intraoperativen Reposition durch die Anlage eines Fixateur externe hilfreich sein, dieser kann in Abhängigkeit von der Knochenqualität zur postoperativen Ruhigstellung belassen oder zum Ende der Operation entfernt werden (Abb.11a-i).

Durch die winkelstabile Verankerung der Schrauben oder Bolzen ist ein Überragen der dorsalen Kortikalis durch die Schraubenspitzen nicht mehr erforderlich, was die Gefahr einer operationsbedingten Schädigung der Strecksehnen ausschließt.

Bei hochgradiger Instabilität des distalen Radiusfragmentes kann die Anwendung einer oder mehrerer interfragmentärer Zugschrauben, meist parallel zur palmaren Fläche des distalen Radius über eine separate Inzision über den Proc. styloideus radii eingebracht, erforderlich werden. Alternativ kann intraoperativtemporär mit Hilfe eines über den Proc. styloideus radii eingebrachter Kirschner-Drahtes zunächst das Instabilitätsausmaß verringert oder zusätzlich von dorsal eine Abstützplatte angelegt werden („Sandwich-Verfahren“) (Abb.12a-d, Abb.13a-c).

Die Operation endet nach Öffnen der Blutsperre mit schichtweisem Wundverschluss über einer Redondrainage, wobei die Fasern des M. pronator quadratus meist nicht adaptiert werden können.

Wichtigster Schritt der Operation ist die subchondrale Platzierung der Schrauben/Bolzen im distalen Fragment.
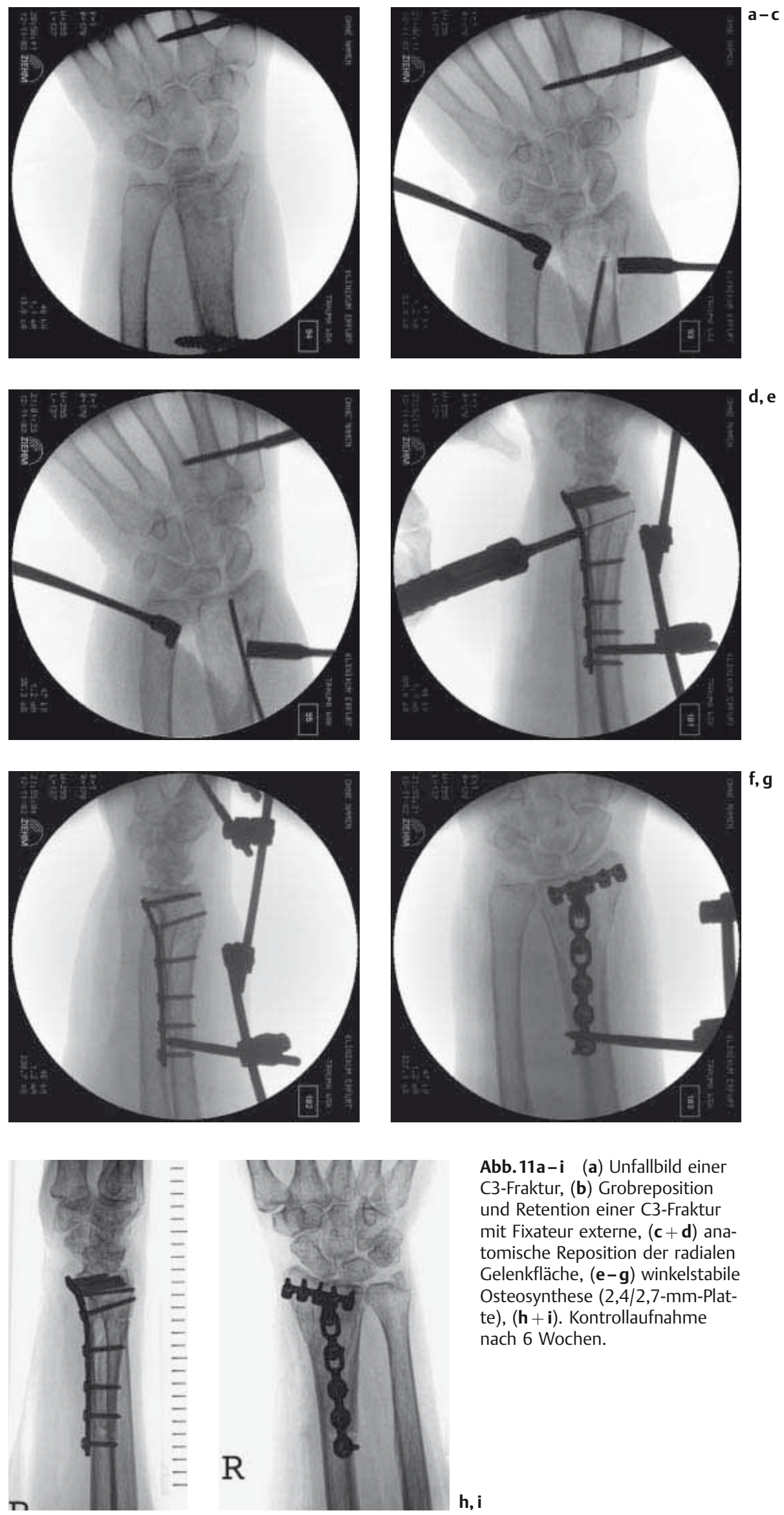

Abb.11a-i (a) Unfallbild einer C3-Fraktur, (b) Grobreposition und Retention einer C3-Fraktur mit Fixateur externe, $(\mathbf{c}+\mathbf{d})$ anatomische Reposition der radialen Gelenkfläche, $(\mathbf{e}-\mathbf{g})$ winkelstabile Osteosynthese (2,4/2,7-mm-Platte), $(\mathbf{h}+\mathbf{i})$. Kontrollaufnahme nach 6 Wochen.

$h, \mathrm{i}$ 
a, b

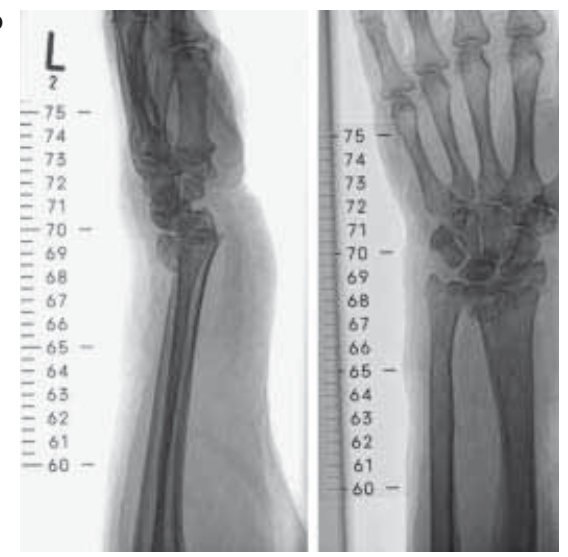

c



d



Abb.12a-d winkelstabile 2,4/2,7-mm-Plattenosteosynthese bei C3-Fraktur mit Fixierung des ulnaren Schlüsselfragments.

\section{Nachbehandlung}

Die von palmar im Sinne der Abstützplattenosteosynthese versorgte Flexionsfraktur des distalen Radius (B3) ist primär übungsstabil und wird lediglich bis zur sicheren Wundheilung auf einer volaren Unterarm-Schiene ruhiggestellt.

Nach Versorgung der distalen Radiusextensionsfraktur in der dargestellten Wei-
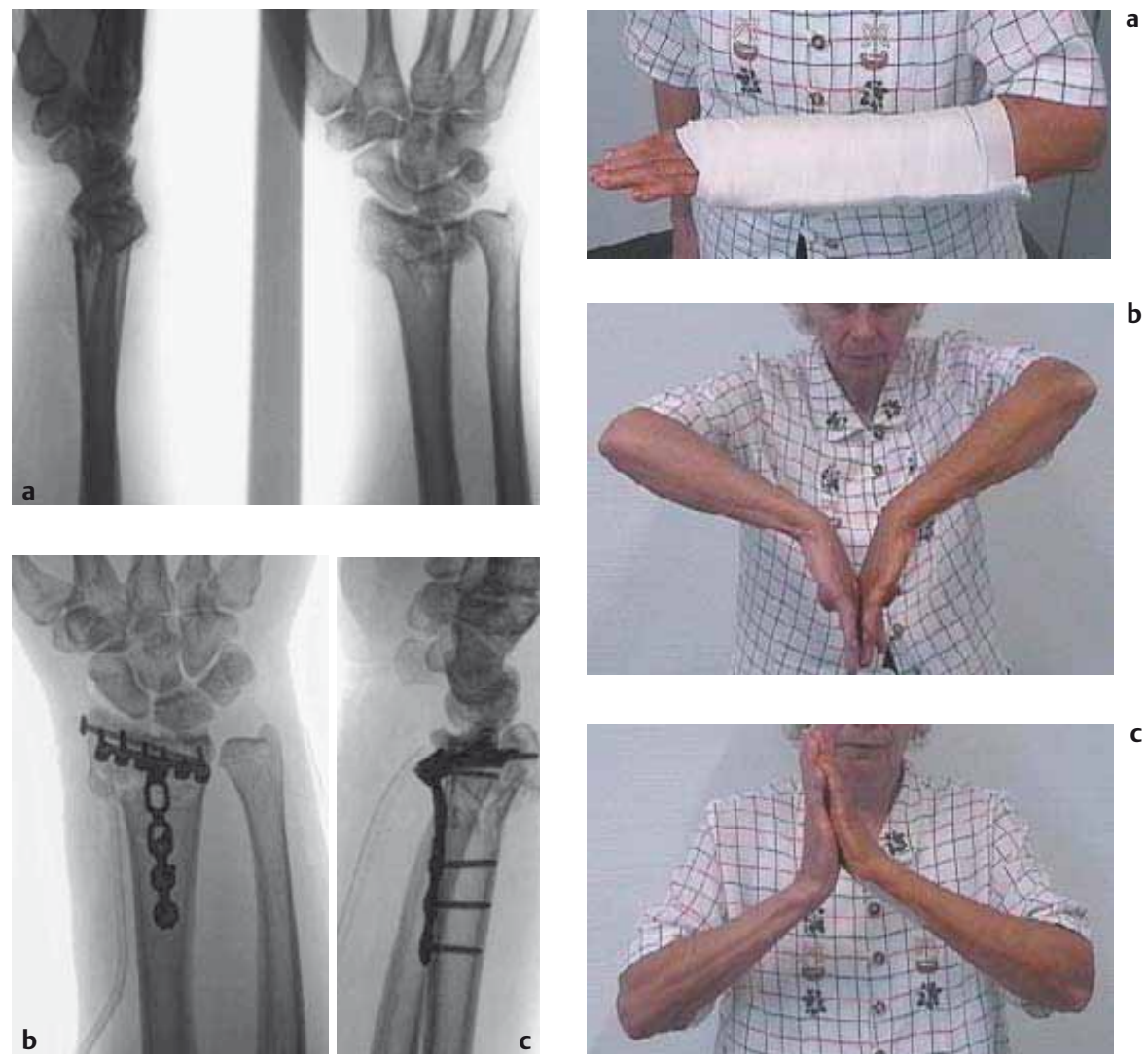

Abb.13a-c 2,4/2,7-mm-Platte mit zusätzl. Schraube.

se mit winkelstabilen Platten von palmar im Sinne einer Zuggurtungsplatte ist dies bei ausreichender Knochenqualität selbst bei höhergradigen Instabilitäten $(C 1-C 2)$ ebenfalls zu erreichen [5]. Lediglich bei extrem hochgradiger Osteoporose und maximaler Instabilität (C3) kann sich in seltenen Fällen eine Phase der Ruhigstellung (meist 2-3 Wochen) anschließen. Dies stellt nach unserer Erfahrung die Ausnahme dar und wird vom Operateur gesondert festgelegt.

Unser Nachbehandlungsschema sieht eine Lagerung der Extremität auf einer volaren Unterarm-Gipsschale bis zur Entfernung des Nahtmaterials vor. Ab dem 1 . postoperativen Tag wird unter krankengymnastischer Anleitung mit der funktionellen Behandlung des Handgelenks und ggf. einer manuellen Lymphdrainage begonnen (Abb.14a-c).

Die Freigabe des Handgelenkes zur Ausübung der Aktivitäten des täglichen Lebens ist bei ausreichender Knochenqualität nach abgeschlossener Wundheilung möglich, eine Maximalbelastung der Hand ist erst bei beginnender knöcherner
Konsolidierung nach Ablauf der 6. postoperativen Woche anzuraten.

Größter Vorteil der palmaren Plattenosteosynthese mit winkelstabilen Implantaten bei instabilen Extensionsfrakturen gegenüber allen anderen Osteosyntheseverfahren ist die Möglichkeit der frühzeitigen Freigabe des Handgelenkes zur Vermeidung einer sympathischen Reflexdystrophie.

\section{Materialentfernung}

Die Entfernung des Osteosynthesematerials nach palmarer Plattenosteosynthese des distalen Radius ist nur sehr selten indiziert. Durch den ausgezeichneten Weichteilmantel an der Beugeseite des Unterarmes treten hier praktisch nie Weichteilprobleme auf. Lediglich $\mathrm{zu}$ lang gewählte Schrauben, die die dorsale Radiuskortikalis überragen, können zu Strecksehnenalterationen bis hin zur Sehnenruptur führen und somit eine Indikation zur Entfernung des Osteosynthesematerials darstellen.

Die Entfernung palmarer Platten bleibt Einzelfällen vorbehalten. 


\section{Ergebnisse}

Im Rahmen einer klinischen und röntgenologischen Nachuntersuchung wurden 73 von 98 Patienten kontrolliert, die von 1/1996-12/1997 eine palmare Plattenosteosynthese mit einer konventionellen - nicht winkelstabilen -3,5-mmT-Platte erhielten [2]. Nach Beurteilung unter Verwendung des Scoring-Systems von Cooney und Bussey wurde das klinische Bild in $88 \%$ der Fälle als "sehr gut“ bis „ausreichend“ und in $12 \%$ als „unzureichend" eingestuft.

Das röntgenologische Ergebnis, bewertet nach dem Score der Arbeitsgruppe „distaler Radius“ der deutschen Sektion der AO, wurde zu $31 \%$ als „gelungen“, bei $59 \%$ als „tolerabel“ und bei $10 \%$ als unzureichend bewertet. Dies betraf bis auf zwei A3-Frakturen ausschließlich C2und C3-Frakturen.

Als Komplikationen wurden zwei Wundinfekte mit notwendigem Verfahrenswechsel und 10 deutliche dorsoaxiale Korrekturverluste mit drei Reosteosynthesen und vier Verfahrenswechsel beobachtet. Intraoperative Komplikationen, revisionsbedürftige Hämatome, posttraumatische Karpaltunnelsyndrome oder Reflexdystrophien wurden nicht beschrieben.

Die gleiche Untersuchung beinhaltete eine Kontrolle von 59 von insgesamt 71 Patienten, die in der Zeit von 1/199812/1999 mit einer winkelstabilen Platte von palmar versorgt worden waren (distale Radius-T-Platte 2,4,/2,7 mm, Synthes). Hier wurde bei $58 \%(\mathrm{n}=34)$ der Patienten ein „sehr gutes“ Resultat, bei $28 \%$ $(n=17)$ ein „gutes“ Ergebnis und bei $14 \%$ $(\mathrm{n}=8)$ ein „ausreichendes“ Ergebnis beobachtet. Ein „unzureichendes“ klinisches Ergebnis ließ sich in keinem Fall nachweisen.

Röntgenologisch zeigte sich nach winkelstabiler palmarer Plattenosteosynthese nach der 6.postoperativen Woche in $68 \%(n=48)$ der Fälle als gelungen und $32 \%(n=23)$ als tolerabel. Unbefriedigende röntgenologische Ergebnisse ließen sich nicht objektivieren.

Als Komplikationen wurden in dieser Gruppe ein postoperatives Karpaltunnelsyndrom und (überwiegend in der Lern- phase) vier fehlimplantierte winkelstabile Schrauben beschrieben, die fünf vorzeitige Materialentfernungen zur Folge hatten. Die Komplikationsrate lag somit bei 7\%. Wundheilungsstörungen, dorsoaxiale Dislokationen oder andere Komplikationen, insbesondere posttraumatische Reflexdystrophien, wurden nicht beobachtet.

\section{Schlussfolgerung}

Die palmare Plattenosteosynthese bei distaler Radiusfraktur ist für die Flexionsfrakturen das Verfahren der Wahl mit der Möglichkeit der sofortigen funktionellen Nachbehandlung.

Durch winkelstabile Implantate mit an die Form des distalen Radius angepasstem Design können auch instabile Extensionsfrakturen mit Schädigung der radialen Gelenkfläche selbst beim osteoporotischen Knochen ohne Korrekturverlust stabil von palmar versorgt und frühfunktionell nachbehandelt werden.

Die Gefahr der sympathischen Reflexdystrophie kann fast vollständig ausgeschlossen werden.

Weitere Vorteile sind ein komfortabler radiopalmarer Zugang, eine breite Implantatpalette mit der Möglichkeit der Erreichung der Winkelstabilität sowie eine geringe peri- und postoperative Komplikationsrate.

Somit könnte die palmare Plattenosteosynthese auch für die Extensionsfrakturen des distalen Radius zum Standardverfahren werden.

Behandlungsschema für Extensionsfrakturen des distalen Radius an der Klinik für Unfallchirurgie am Helios-Klinikum Erfurt

- UA-Gips im „Mädchenfänger“

- OP. vom 0.-7. posttraumatischen Tag

- Drainage am 2.p.o. Tag ex

- frühfunktionelle Beübung ab 3. postoperativem Tag

- volare Schiene bis Fadenzug

- Entlassung bei Monoverletzten $<7 d$

\section{Literatur}

1 Colles A. On the fracture of the carpal extremity of radius. Edinburgh Med Surg J 1814; 10: $182-186$

2 Uzdil T, Neumann W, Bauschke A, Winker KH. Die palmare winkelstabile Plattenosteosynthese bei distalen Radiusextensionsfrakturen Akt. Traumatologie 2001; 31: 141 148

3 Wiemer P et al.. Frakturen am distalen Radius - Wandel der therapeutischen Strategien. Orthopäde 1999; 28: 846-852

4 Zimmermann P et al.. Distale, metaphysäre Kompressionsfrakturen des Radius - Ergebnisse nach offener Reposition, stabiler Defektauffüllung mit einem kortikospongiösen Beckenspan und Plattenosteosynthese. Der Unfallchirurg 1998; 101: $762-768$

5 Schmidt J, Petereit U, Winker KH. Die volare Plattenosteosynthese als Zuggurtungsprinzip bei der Extensionsfraktur des distalen Radius. Akt Traumatologie 1997; 27: 23 -25

\section{Torsten Pabst}

Facharzt für Chirurgie

Dr. med. Torsten Uzdil

Facharzt für Chirurgie

Prof. Dr. med. Karl Heinrich Winker Chefarzt

Klinik für Unfall-, Hand- und Wiederherstellungschirurgie HELIOS-Klinikum Erfurt Nordhäuser Str. 74 D-99089 Erfurt 